TO APPEAR IN APJ LETTERS

Preprint typeset using LTEX style emulateapj v. 08/22/09

\title{
CORRECTING STELLAR OSCILLATION FREQUENCIES FOR NEAR-SURFACE EFFECTS
}

\author{
HANS KJELDSEN $^{1}$, TIMOTHY R. BEDDING ${ }^{2}$ AND JøRGEN CHRISTENSEN-DALSGAARD ${ }^{1}$ \\ To appear in ApJ Letters
}

\begin{abstract}
In helioseismology, there is a well-known offset between observed and computed oscillation frequencies. This offset is known to arise from improper modeling of the near-surface layers of the Sun, and a similar effect must occur for models of other stars. Such an effect impedes progress in asteroseismology, which involves comparing observed oscillation frequencies with those calculated from theoretical models. Here, we use data for the Sun to derive an empirical correction for the near-surface offset, which we then apply three other stars ( $\alpha$ Cen A, $\alpha$ Cen B and $\beta$ Hyi). The method appears to give good results, in particular providing an accurate estimate of the mean density of each star.
\end{abstract}

Subject headings: stars: individual ( $\beta$ Hyi, $\alpha$ Cen A, $\alpha$ Cen B) — stars: oscillations — Sun: helioseismology

\section{INTRODUCTION}

Both helio- and asteroseismology involve comparing observed oscillation frequencies with those calculated from theoretical models. However, for the Sun there is a longstanding systematic offset between observed and computed frequencies that is known to arise from improper modeling of the near-surface layers (Christensen-Dalsgaard et al. 1988; Dziembowski et al. 1988; Christensen-Dalsgaard et al. 1996; Christensen-Dalsgaard \& Thompson 1997). This offset is independent of the angular degree of the mode $(l)$ and increases with frequency. A similar offset must occur for models of other stars, and should be taken into account whenever observations of stellar oscillations are compared with theory. In this Letter we use data for the Sun to derive an empirical correction for these near-surface effects and show how to apply this to other stars. The method appears to give good results, in particular providing an accurate estimate of the mean density of each star.

\section{METHOD}

The p-mode oscillations in solar-type stars for a given angular degree $l$ are approximately equally spaced in frequency, with a separation of $\Delta \nu$ (the so-called large separation; see Christensen-Dalsgaard 2004 for a review of the theory of solar-like oscillations). Since the offset from incorrect modelling of the near-surface layers is independent of $l$, we can derive the correction by considering only the radial modes $(l=0)$ and then apply it to all modes.

Suppose we have a set of observed frequencies for radial modes, $\nu_{\text {obs }}(n)$, where $n$ is the radial order. Suppose also that $\nu_{\text {best }}(n)$ are the frequencies from the best model, by which we mean the one that best describes the parameters and internal structure of the star, but which still fails to model correctly the surface layers,

For the Sun, the difference between observed and best model frequencies turns out to be well fitted by a power law, which has the convenient property of being free of a frequency

\footnotetext{
${ }^{1}$ Danish AsteroSeismology Centre (DASC), Department of Physics and Astronomy, University of Aarhus, DK-8000 Aarhus C, Denmark; hans@phys.au.dk,jcd@phys.au.dk

${ }^{2}$ Institute of Astronomy, School of Physics, University of Sydney, NSW 2006, Australia; bedding@physics.usyd.edu.au
}

scale (see also Christensen-Dalsgaard \& Gough 1980):

$$
\nu_{\mathrm{obs}}(n)-\nu_{\text {best }}(n)=a\left(\frac{\nu_{\mathrm{obs}}(n)}{\nu_{0}}\right)^{b},
$$

where $\nu_{0}$ is a suitably chosen reference frequency, and $a$ and $b$ are parameters to be determined.

Suppose further that we have calculated a reference model that has frequencies $\nu_{\text {ref }}(n)$ and is close to the best model. From homology scaling it then follows that, to a good approximation,

$$
\nu_{\text {best }}(n)=r \nu_{\text {ref }}(n),
$$

where the scaling factor $r$ is related to the mean densities $\bar{\rho}_{\text {best }}$ and $\bar{\rho}_{\text {ref }}$ of the best and reference models by

$$
\bar{\rho}_{\text {best }}=r^{2} \bar{\rho}_{\text {ref }} \text {. }
$$

Given a determination of $r$, equation (3) provides our best estimate of the mean density of the star.

Substituting equation (2) into equation (1) gives

$$
\nu_{\mathrm{obs}}(n)-r \nu_{\mathrm{ref}}(n)=a\left(\frac{\nu_{\mathrm{obs}}(n)}{\nu_{0}}\right)^{b}
$$

and differentiating with respect to $n$ gives

$$
\Delta \nu_{\mathrm{obs}}(n)-r \Delta \nu_{\mathrm{ref}}(n)=a b\left(\frac{\nu_{\mathrm{obs}}(n)}{\nu_{0}}\right)^{b-1} \frac{\Delta \nu_{\mathrm{obs}}(n)}{\nu_{0}} .
$$

Combining and rearranging these last two equations gives

$$
r=(b-1)\left(b \frac{\nu_{\mathrm{ref}}(n)}{\nu_{\mathrm{obs}}(n)}-\frac{\Delta \nu_{\mathrm{ref}}(n)}{\Delta \nu_{\mathrm{obs}}(n)}\right)^{-1}
$$

and

$$
b=\left(r \frac{\Delta \nu_{\mathrm{ref}}(n)}{\Delta \nu_{\mathrm{obs}}(n)}-1\right)\left(r \frac{\nu_{\mathrm{ref}}(n)}{\nu_{\mathrm{obs}}(n)}-1\right)^{-1} .
$$

If we know $b$ then we can calculate $r$ using equation (6), or vice versa using equation (7). We can then obtain $a$ using equation (4).

We now show how to apply the above method to a set of observed and calculated frequencies. Suppose we have frequencies for $N$ radial modes with orders $n_{1}, n_{2}, \ldots, n_{N}$ (not necessarily consecutive). We use these to calculate the four terms needed to evaluate equation (6) or (7). For $\nu_{\mathrm{obs}}(n)$ and 
$\nu_{\text {ref }}(n)$, we simply use the means of the given sets of frequencies, which we denote by $\left\langle\nu_{\text {obs }}(n)\right\rangle$ and $\left\langle\nu_{\text {ref }}(n)\right\rangle$.

To estimate the large separations, we calculate the slope of a linear least-squares fit to the given frequencies (as a function of $n$ ):

$$
\begin{aligned}
\left\langle\Delta \nu_{\mathrm{obs}}(n)\right\rangle & =\frac{\sum_{i=1}^{N}\left(\nu_{\mathrm{obs}}\left(n_{i}\right)-\left\langle\nu_{\mathrm{obs}}(n)\right\rangle\right)\left(n_{i}-\langle n\rangle\right)}{\sum_{i=1}^{N}\left(n_{i}-\langle n\rangle\right)^{2}} \\
\left\langle\Delta \nu_{\mathrm{ref}}(n)\right\rangle & =\frac{\sum_{i=1}^{N}\left(\nu_{\mathrm{ref}}\left(n_{i}\right)-\left\langle\nu_{\mathrm{ref}}(n)\right\rangle\right)\left(n_{i}-\langle n\rangle\right)}{\sum_{i=1}^{N}\left(n_{i}-\langle n\rangle\right)^{2}} .
\end{aligned}
$$

We must then assume a value for either $b$ or $r$, and use equation (6) or (7) to estimate the other. Finally, the value of $a$ is found from equation (4), as follows:

$$
a=\frac{\left\langle\nu_{\mathrm{obs}}(n)\right\rangle-r\left\langle\nu_{\mathrm{ref}}(n)\right\rangle}{N^{-1} \sum_{i=1}^{N}\left(\nu_{\mathrm{obs}}\left(n_{i}\right) / \nu_{0}\right)^{b}} .
$$

We now proceed to apply this method to the Sun in order to measure $b(\$ 3)$, and then adopt this value of $b$ for other stars (\$4).

\section{APPLICATION TO THE SUN}

For the Sun we took Model $\mathrm{S}$ of Christensen-Dalsgaard et al. (1996), as listed in the first row of Table 1 We assumed this to be the "best" solar model, in the sense defined above, which means we can set $r=1$ (see equation 2). For the observed solar frequencies, we used those measured by Lazrek et al. (1997) with the GOLF instrument on the SOHO spacecraft.

We followed the procedure described above, choosing $\nu_{0}=$ $3100 \mu \mathrm{Hz}$ and setting $r=1$, and using the data to measure $b$ and $a$. We have chosen to use the nine modes centred at the peak of the oscillation power, from which we obtained the results shown in the first line of Table 2 and a value of $b=4.90$. The differences between observed and Model $\mathrm{S}$ frequencies are plotted as the squares in Fig. 1, and the solid curve is the function given by equation (1).

The above fit was made for the strongest 9 radial modes in the Sun. We repeated the analysis for different numbers of modes (all values from 7 to 13), and found the derived value of $b$ to range from 4.4 to 5.25 . Clearly, the frequency differences do not exactly follow a power law, and so the exponent in the power-law fit depends substantially on the frequency range. Importantly, the value for $a$ varies by less than $0.1 \mu \mathrm{Hz}$ in all cases.

In addition to Model $\mathrm{S}$, we also considered models denoted $\mathrm{S}^{-}$and $\mathrm{S}^{+}$from the same evolution sequence as Model S, but with ages of 2.25 and $7.44 \mathrm{Gyr}$, respectively. The parameters of these models are given in Table 1. We kept $b$ fixed at the value found for the "best" model (4.90) and used equation (6) to estimate $r$. The results are shown in Table 2 and also in Fig. 1. Importantly, the derived density of the Sun $\left(r^{2} \bar{\rho}_{\text {ref }}\right)$ is correct for both these calculations, despite the very different densities of the models themselves, giving us confidence that the method has been successful. Even more importantly, the derived density of the Sun is completely insensitive to the choice of $b$. This reflects the fact that the value of $r$ obtained by fitting to equation 4 is not sensitive to the exact form of the function on the right-hand side, so long as that function tends to zero with decreasing frequency.

4. APPLICATION TO $\alpha$ Cen A, $\alpha$ Cen B AND $\beta$ Hyi
We have considered three stars for which observations and models have been published. For $\alpha$ Cen A we took observed frequencies (radial modes only) from four sources: Bouchy \& Carrier (2002), Bedding et al. (2004), Bazot et al. (2007) and Fletcher et al. (2006). This gave a set of 33 measured frequencies for 11 orders, all of which were given equal weight in the fitting process. For $\alpha$ Cen B the observed frequencies were taken from Kieldsen et al. (2005) and those for $\beta$ Hyi from Bedding et al. (2007). In each case, we used all the detected $l=0$ frequencies.

The models that we have used for these stars are listed in Table 1. These include published models of $\alpha$ Cen A and B by Morel et al. (2000) and Thévenin et al. (2002), of $\alpha$ Cen B by Tang et al. (2008), and of $\beta$ Hyi by Fernandes \& Monteiro (2003). In addition to published models, we have considered several computed with the Aarhus stellar evolution code (ASTEC, Christensen-Dalsgaard 2008). Models A and B are models of the $\alpha$ Cen system, computed with essentially the same physics as Model S and fitted (T. Teixeira et al. 2008, in preparation) to the observations of Bedding et al. (2004) and Kjeldsen et al. (2005). For $\beta$ Hyi, Model H matches the parameters reported by North et al. (2007) and was computed with similar physics, but neglecting diffusion and settling. Finally, Models $\mathrm{H}^{-}$and $\mathrm{H}^{+}$are from the same evolution sequence as Model $\mathrm{H}$, but with substantially different ages and densities, which bracket those of Model $\mathrm{H}$.

For each model we used the value of $b$ found for the Sun, and used equation (6) to estimate $r$. The results are shown in Table 2, and also in Figs. 2, 4 . Note that there is considerable scatter in the observed frequencies of $\alpha$ Cen $\mathrm{A}$ and $\mathrm{B}$ due to the relatively short span of the observations relative to the mode lifetime. Taking this into account, it is once again encouraging to see that the power law (with a single value of $b$ ) provides a good fit to the frequency differences, and that for each star there is good agreement between the densities derived from the different models.

To estimate uncertainties, we have repeated the fits for the same range of $b$ values considered in $\$ 3(4.4-5.25)$ and again found that there is no effect on the calculated density, to the precision quoted in Table 2 . Over this range, the change in $a$ is less than $1 \mu \mathrm{Hz}$ for $\beta \mathrm{Hyi}$, less than $0.4 \mu \mathrm{Hz}$ for $\alpha$ Cen $\mathrm{A}$ and less than $0.7 \mu \mathrm{Hz}$ for $\alpha$ Cen B. The changes in the correction terms plotted in Figs. 24 4 are comparable or slightly bigger.

For each star, we can identify the model that is closest to being the "best" model as the one having $r$ closest to unity (note this is not necessarily the model with the smallest near-surface offset). These give our best estimate of the stellar density for each star.

North et al. (2007) recently used interferometry to measure the angular diameter of $\beta$ Hyi to be $2.257 \pm 0.019$ mas. They combined this with the parallax (from Hipparcos) and the mean density (from asteroseismology) to determine the radius and mass of the star. Using our best estimate of the mean density of $\beta$ Hyi $\left(0.258 \pm 0.001 \mathrm{~g} \mathrm{~cm}^{-3}\right)$ and the revised Hipparcos parallax (134.070 \pm 0.110 mas; van Leeuwen 2007), we derive slightly updated values, finding a radius of $1.809 \pm 0.015 R_{\odot}(0.85 \%)$ and a mass of $1.085 \pm 0.028 M_{\odot}$ $(2.6 \%)$.

\section{DISCUSSION AND CONCLUSIONS}

The method outlined here for correcting near-surface effects can be applied to model frequencies before they are compared with observations. As in the case of the Sun, we expect that the correction is independent of degree at a given fre- 
quency, for low-degree acoustic modes, and thus the correction determined from radial modes can be applied to all such modes.

There is, however, an important exception. In evolved stars, mixed modes may be observed that have the character of gravity modes in the deep interior of the star. Observational evidence for mixed modes has been found in $\eta$ Boo (Kjeldsen et al. 1995, 2003; Carrier et al. 2005) and $\beta$ Hyi (Bedding et al. 2007). Owing to the larger amplitude of these modes in the stellar interior and hence their higher inertia, their frequencies are less affected by the surface effects. Techniques need to be developed to take this into account in the application of the surface correction before the frequencies are analyzed.

The use of a single power law is made plausible by the knowledge that the offsets we are modelling are caused by the properties of the near-surface layers and hence presumably depend only on surface gravity, effective temperature and composition, and not on the details of the internal properties of the star. This may be particularly true of the exponent which, in the simple analysis by Christensen-Dalsgaard \& Gough (1980), is determined by an effective average polytropic index (i.e., the relation between pressure and density) in the near-surface layers.

It is important to note that, owing to its strong frequency dependence, the offset also affects the large frequency sepa- ration $\Delta \nu$, as is indeed implicit in equation (5). For example, although Model $\mathrm{S}$ is one of the best available models of the Sun, it has a large separation that is $1 \mu \mathrm{Hz}$ greater than the observed value. Thus, attempting to fit to stellar models based on $\Delta \nu$ will introduce systematic errors, unless the corresponding correction is applied.

We have shown how to identify the model for each star that is closest to the "best" model, by requiring that $r$ be as close as possible to unity. This gives us an extremely accurate estimate of the mean stellar density. However, it is important to point out that, while a model with $r$ close to unity gives a good match to the overall structure of the star, it does not necessarily reproduce the structure of the core or give a reliable estimate of the stellar age. Determination of those properties requires taking into account the frequencies of the non-radial modes (including the small frequency separations). That is the next stage of model fitting, which can be done after the surface correction has been applied to all modes.

We thank Teresa Teixeira for assistance in determining Models A and B, and Mario Monteiro for providing the frequencies of Model $S_{0}$ for $\beta$ Hyi in electronic form. This work was supported financially by the Danish Natural Science Research Council and the Australian Research Council.

\section{REFERENCES}

Bazot, M., Bouchy, F., Kjeldsen, H., Charpinet, S., Laymand, M., \& Vauclair, S., 2007, A\&A, 470, 295.

Bedding, T. R., Kjeldsen, H., Arentoft, T., et al., 2007, ApJ, 663, 1315.

Bedding, T. R., Kjeldsen, H., Butler, R. P., et al., 2004, ApJ, 614, 380.

Bouchy, F., \& Carrier, F., 2002, A\&A, 390, 205.

Carrier, F., Eggenberger, P., \& Bouchy, F., 2005, A\&A, 434, 1085.

Christensen-Dalsgaard, J., 2004, Sol. Phys., 220, 137.

Christensen-Dalsgaard, J., 2008, Ap\&SS. in press (astro-ph/0710.3114).

Christensen-Dalsgaard, J., Däppen, W., Ajukov, S. V., et al., 1996, Sci, 272, 1286.

Christensen-Dalsgaard, J., Däppen, W., \& Lebreton, Y., 1988, Nat, 336, 634. Christensen-Dalsgaard, J., \& Gough, D. O., 1980, Nat, 288, 544.

Christensen-Dalsgaard, J., \& Thompson, M. J., 1997, MNRAS, 284, 527.

Dziembowski, W. A., Paternó, L., \& Ventura, R., 1988, A\&A, 200, 213.

Fernandes, J., \& Monteiro, M. J. P. F. G., 2003, A\&A, 399, 243.

Fletcher, S. T., Chaplin, W. J., Elsworth, Y., Schou, J., \& Buzasi, D., 2006, MNRAS, 371, 935.
Kjeldsen, H., Bedding, T. R., Baldry, I. K., et al., 2003, AJ, 126, 1483. Kjeldsen, H., Bedding, T. R., Butler, R. P., et al., 2005, ApJ, 635, 1281. Kjeldsen, H., Bedding, T. R., Viskum, M., \& Frandsen, S., 1995, AJ, 109, 1313.

Lazrek, M., Baudin, F., Bertello, L., et al., 1997, Sol. Phys., 175, 227.

Morel, P., Provost, J., Lebreton, Y., Thévenin, F., \& Berthomieu, G., 2000, A\&A, 363, 675.

North, J. R., Davis, J., Bedding, T. R., et al., 2007, MNRAS, 380, L83.

Tang, Y.-K., Bi, S.-L., Gai, N., \& Xu, H.-Y., 2008, Chin. J. A\&A. in press.

Thévenin, F., Provost, J., Morel, P., Berthomieu, G., Bouchy, F., \& Carrier, F., 2002, A\&A, 392, L9.

van Leeuwen, F. Hipparcos, the New Reduction of the Raw Data. Springer:

Dordrecht, 2007. 
TABLE 1

DETAILS OF THEORETICAL MODELS

\begin{tabular}{|c|c|c|c|c|c|c|}
\hline Model ID & Star & Mass & Radius & Luminosity & Age (Gyr) & Reference \\
\hline Model S & Sun & 1.000 & 1.000 & 1.000 & 4.52 & Christensen-Dalsgaard et al. (1996) \\
\hline Model S ${ }^{-}$ & ... & 1.000 & 0.930 & 1.286 & 2.25 & this paper \\
\hline Model $\mathrm{S}^{+}$ & & 1.000 & 1.128 & 0.832 & 7.44 & this paper \\
\hline FM2003 & $\beta$ Hyi & 1.100 & 1.899 & 3.540 & 6.82 & Model $S_{0}$ from Fernandes \& Monteiro (2003) \\
\hline Model H & $\beta$ Hyi & 1.080 & 1.818 & 3.539 & 6.09 & this paper \\
\hline Model $\mathrm{H}^{-}$ & $\ldots$ & 1.080 & 1.667 & 3.248 & 5.79 & this paper \\
\hline Model $\mathrm{H}^{+}$ & $\ldots$ & 1.080 & 2.007 & 3.617 & 6.33 & this paper \\
\hline Model A & $\alpha$ Cen A & 1.111 & 1.224 & 1.5460 & 6.96 & this paper \\
\hline M2000 A & $\alpha$ Cen A & 1.160 & 1.228 & 1.527 & 2.71 & Model $A_{B V}$ from Morel et al. (2000) \\
\hline Th2002 A & $\alpha$ Cen A & 1.100 & 1.230 & 1.519 & 4.85 & Thévenin et al. (2002) \\
\hline Model B & $\alpha$ Cen B & 0.928 & 0.867 & 0.5025 & 6.88 & this paper \\
\hline M2000 B & $\alpha$ Cen B & 0.970 & 0.909 & 0.571 & 2.71 & Model $\mathrm{B}_{\mathrm{BV}}$ from Morel et al. (2000) \\
\hline Th2002 B & $\alpha$ Cen B & 0.907 & 0.857 & 0.5002 & 4.85 & Thévenin et al. (2002) \\
\hline T2008 & $\alpha$ Cen B & 0.929 & 0.869 & 0.4991 & 5.86 & Model M2 from Tang et al. (2008) \\
\hline
\end{tabular}

TABLE 2

NEAR-SURFACE CORRECTIONS

\begin{tabular}{|c|c|c|c|c|c|c|c|c|c|}
\hline Model & $n_{i}$ & $\begin{array}{c}\left\langle\nu_{\mathrm{obs}}(n)\right\rangle \\
(\mu \mathrm{Hz})\end{array}$ & $\begin{array}{c}\left\langle\Delta \nu_{\mathrm{obs}}(n)\right\rangle \\
(\mu \mathrm{Hz})\end{array}$ & $\begin{array}{c}\left\langle\nu_{\mathrm{ref}}(n)\right\rangle \\
(\mu \mathrm{Hz})\end{array}$ & $\begin{array}{c}\left\langle\Delta \nu_{\text {ref }}(n)\right\rangle \\
(\mu \mathrm{Hz})\end{array}$ & $\begin{array}{c}a \\
(\mu \mathrm{Hz})\end{array}$ & $r$ & $\begin{array}{c}\bar{\rho}_{\text {ref }} \\
\left(\mathrm{g} \mathrm{cm}^{-3}\right)\end{array}$ & $\begin{array}{c}r^{2} \bar{\rho}_{\text {ref }} \\
\left(\mathrm{g} \mathrm{cm}^{-3}\right)\end{array}$ \\
\hline \multicolumn{10}{|c|}{ Sun $\left(\nu_{0}=3100 \mu \mathrm{Hz}\right)$} \\
\hline Model S & $17-25$ & 3034.15 & 134.810 & 3038.95 & 135.854 & -4.73 & 1.00000 & 1.408 & 1.408 \\
\hline Model S ${ }^{-}$ & $17-25$ & 3034.15 & 134.810 & 3386.40 & 150.761 & -1.54 & 0.89644 & 1.748 & 1.405 \\
\hline Model S ${ }^{+}$ & $17-25$ & 3034.15 & 134.810 & 2540.67 & 114.155 & -8.67 & 1.19770 & 0.982 & 1.408 \\
\hline \multicolumn{10}{|c|}{$\beta$ Hyi $\left(\nu_{0}=1000 \mu \mathrm{Hz}\right)$} \\
\hline Model $\mathrm{H}^{-}$ & $13-19$ & 1004.42 & 57.244 & 1134.75 & 65.985 & -4.57 & 0.88978 & 0.329 & 0.260 \\
\hline Model H & $13-19$ & 1004.42 & 57.244 & 1001.90 & 58.417 & -5.19 & 1.00847 & 0.253 & 0.258 \\
\hline FM2003 & $13-19$ & 1004.42 & 57.244 & 948.58 & 55.714 & -6.90 & 1.06723 & 0.226 & 0.258 \\
\hline Model $\mathrm{H}^{+}$ & $13-19$ & 1004.42 & 57.244 & 868.28 & 50.654 & -5.32 & 1.16385 & 0.188 & 0.255 \\
\hline \multicolumn{10}{|c|}{$\alpha$ Cen $\mathrm{A}\left(\nu_{0}=2400 \mu \mathrm{Hz}\right)$} \\
\hline Model S ${ }^{+}$ & $16-26$ & 2335.89 & 105.541 & 2509.96 & 114.026 & -3.18 & 0.93196 & 0.982 & 0.853 \\
\hline M2000 A & $16-26$ & 2335.89 & 105.541 & 2362.88 & 107.949 & -6.48 & 0.99141 & 0.882 & 0.867 \\
\hline Model A & $16-26$ & 2335.89 & 105.541 & 2345.37 & 106.344 & -2.05 & 0.99686 & 0.854 & 0.849 \\
\hline Th2002 A & $16-26$ & 2335.89 & 105.541 & 2303.36 & 104.927 & -4.78 & 1.01626 & 0.833 & 0.860 \\
\hline \multicolumn{10}{|c|}{$\alpha \operatorname{Cen~B~}\left(\nu_{0}=4100 \mu \mathrm{Hz}\right)$} \\
\hline Th2002 B & $19-27$ & 3890.70 & 161.482 & 3910.71 & 162.605 & -2.11 & 0.99534 & 2.030 & 2.011 \\
\hline Model B & 19-32 & 4261.05 & 161.988 & 4274.09 & 162.913 & -1.87 & 0.99762 & 2.009 & 1.999 \\
\hline T2008 & 19-32 & 4261.05 & 161.988 & 4261.92 & 162.697 & -2.96 & 1.00087 & 1.995 & 1.998 \\
\hline M2000 B & $19-32$ & 4261.05 & 161.988 & 4064.00 & 155.400 & -4.15 & 1.05006 & 1.819 & 2.006 \\
\hline Model S- & $19-32$ & 4261.05 & 161.988 & 3979.80 & 152.166 & -4.08 & 1.07225 & 1.748 & 2.010 \\
\hline
\end{tabular}




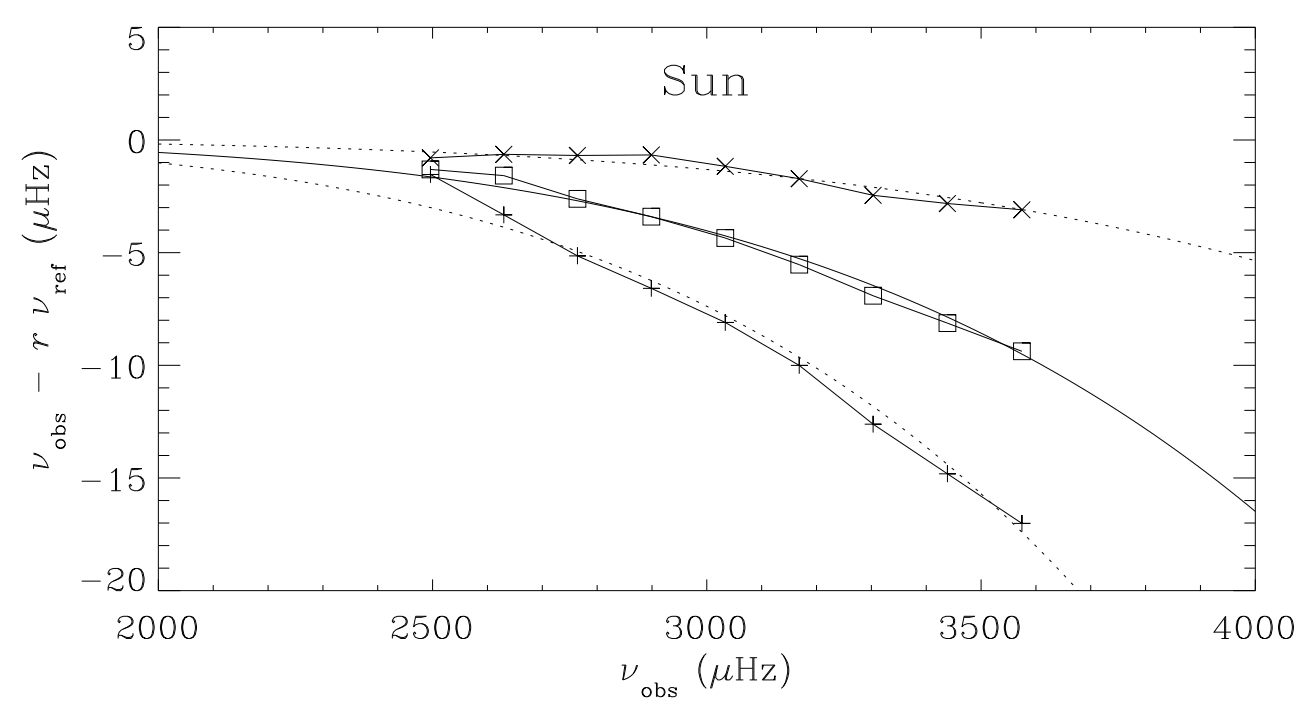

FIG. 1.- The difference between observed and calculated frequencies for radial modes in the Sun. The squares are for Model S, with the solid curve showing a fit to equation (4) with $r=1$, which gives $b=4.90$ (see \$3). Also shown are the results of applying the same corrections to Model $\mathrm{S}^{-}$(crosses) and Model $\mathrm{S}^{+}$ (pluses). The dotted curves show the corrections calculated from equation 4.

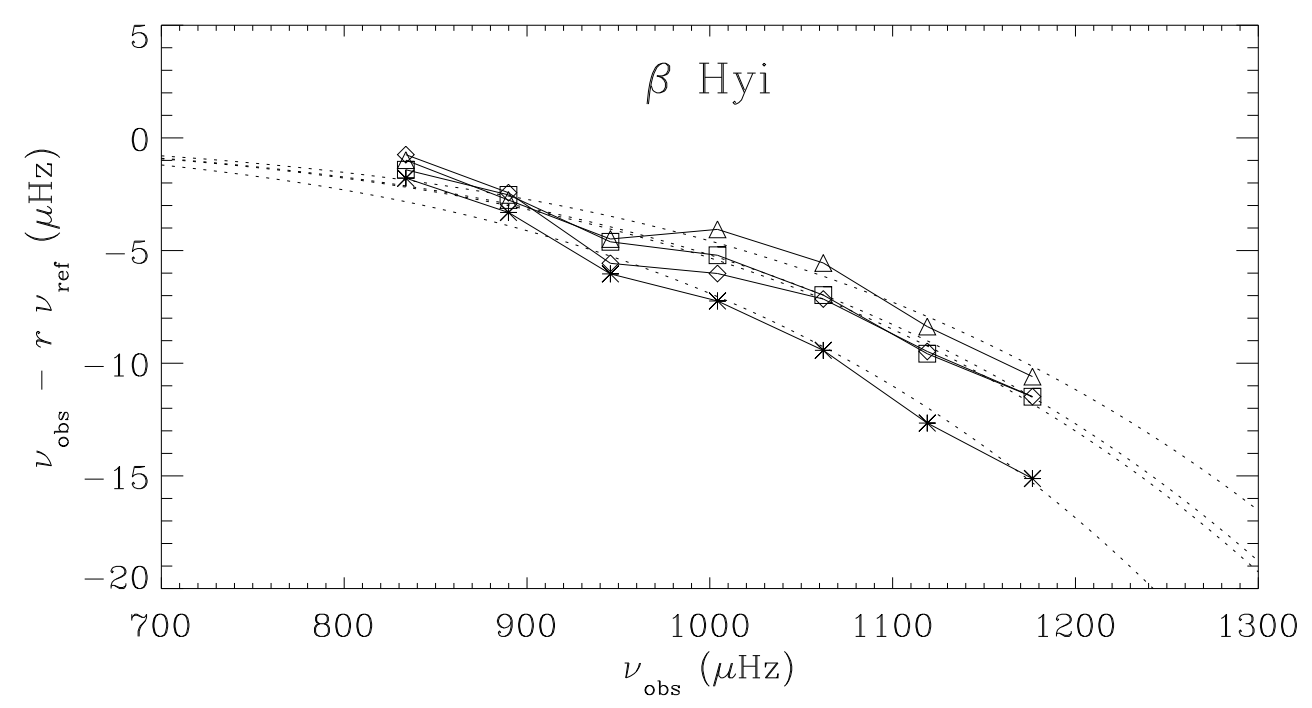

FIG. 2.- The difference between observed and calculated frequencies for radial modes in $\beta$ Hyi. The models shown are: Model H (squares), Model $\mathrm{H}^{-}$ (triangles), Model $\mathrm{H}^{+}$(diamonds) and FM2003 (asterisks). The dotted curves show the corrections calculated from equation (4). 


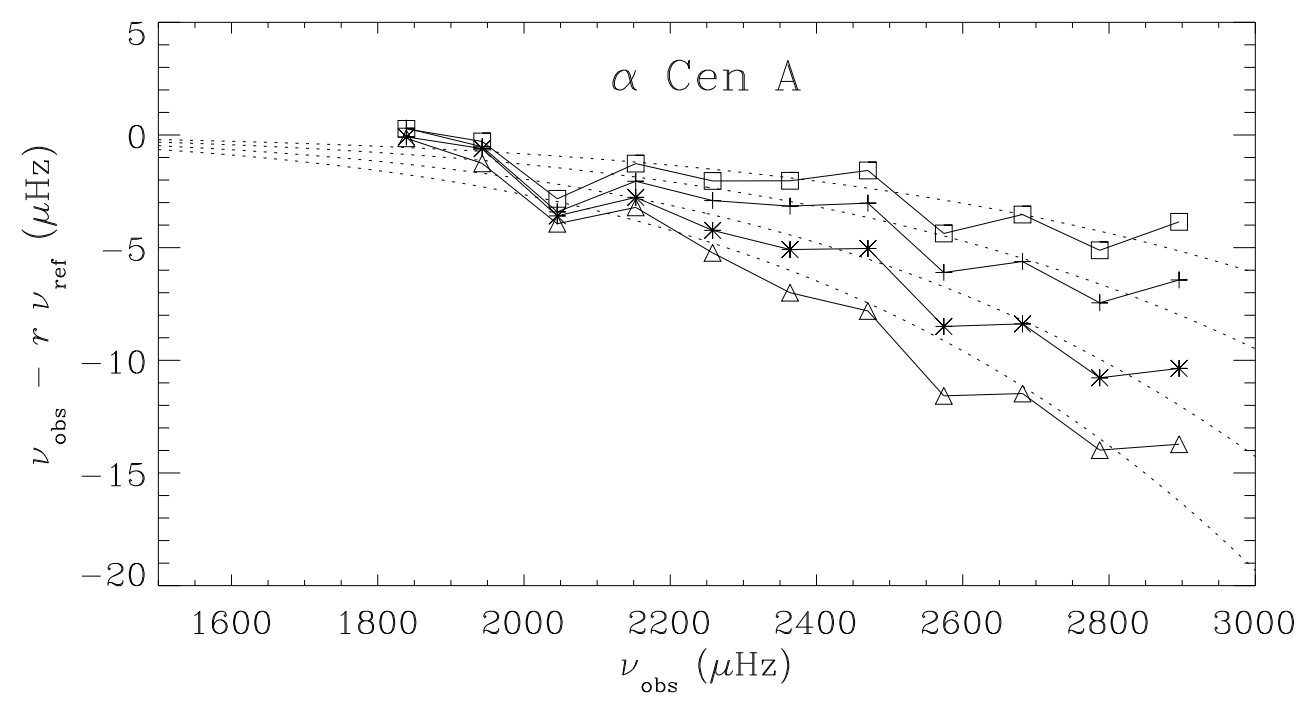

FIg. 3. - Same as Fig. 2 but for $\alpha$ Cen A. The models shown are: Model A (squares), M2000 A (triangles), Model S ${ }^{+}$(pluses) and Th2002 A (asterisks).

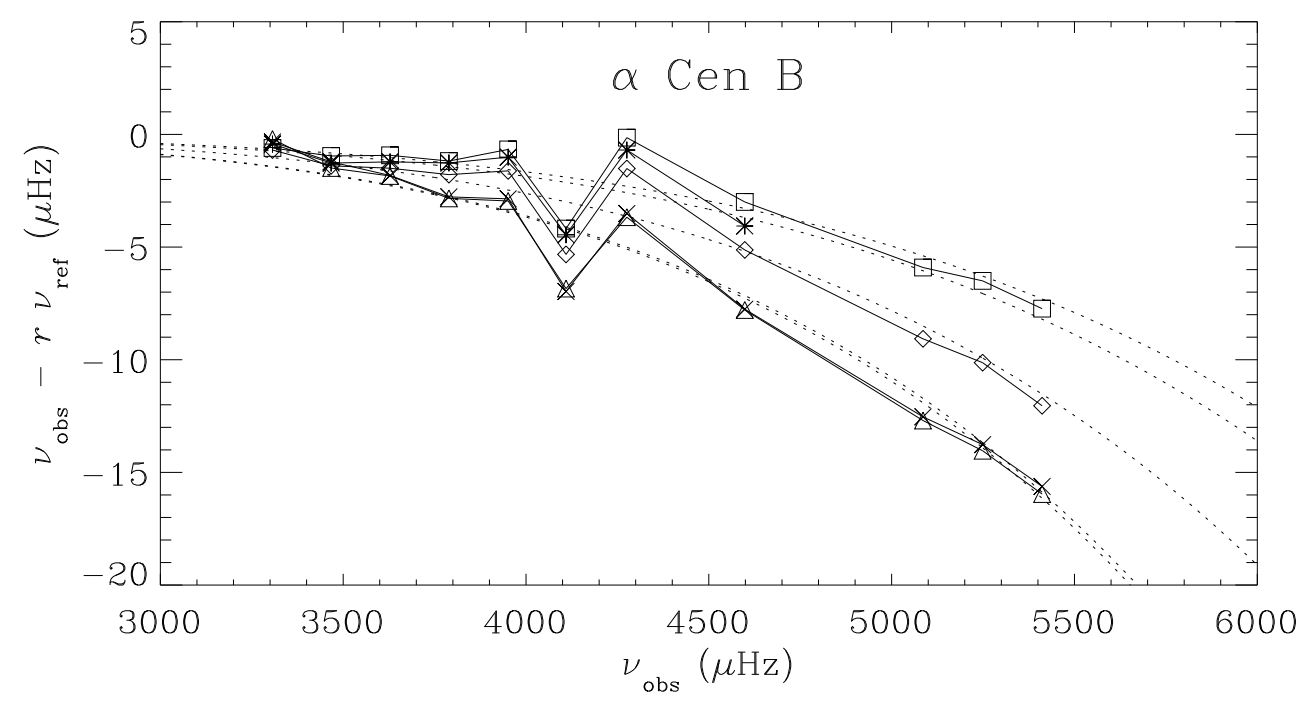

FIG. 4.- Same as Fig. 2 but for $\alpha$ Cen B. The models shown are: Model B (squares), M2000 B (triangles), Model S- (crosses), Th2002 B (asterisks) and T2008 (diamonds). 